www.jmscr.igmpublication.org

Index Copernicus Value: 79.54

ISSN (e)-2347-176x ISSN (p) 2455-0450

crossref DOI: https://dx.doi.org/10.18535/jmscr/v7i4.154

\title{
Analysis of Prescription Pattern and Impact of Patient Counselling in the Quality of life of Patients with Coronary Artery Disease: A Pilot Study
}

\author{
Authors \\ Shamna $C^{1}$, Neenu Babu ${ }^{1}$, Sreelekshmi V S ${ }^{1}$, Philip John Sebastian ${ }^{1}$, \\ Nithin Manohar $R^{2}$, Prasobh G Nair ${ }^{3}$, I John wesely ${ }^{4}$ \\ ${ }^{1}$ Pharm-D Students, ${ }^{2}$ Assistant Professor of Department of Pharmacy Practice \\ ${ }^{3}$ Principal, ${ }^{4} \mathrm{HOD}$ of Dept of Pharmacy Practice \\ Sreekrishna College of Pharmacy and Research Centre, Trivandrum, Kerala, India \\ *Corresponding Author \\ Nithin Manohar R \\ Email: ntnmanohar@yahoo.com
}

\begin{abstract}
Background: Recently it has been found that cardiovascular disease is the most frequent cause of morbidity and mortality throughout the world. The objective of present study was to evaluate the different existing drug treatment strategies used in the management of coronary artery diseases and counsel the patients according to their modifiable risk factors and assess their quality of life.

Method: This was a prospective observational study conducted in the department of cardiology. A total of 25 patients presenting with varied category of CAD were screened and analyzed. The quality of life of patients were analyzed by Short form 36 (SF-36) Questionnaire.

Results: In this study, among 25 cases of coronary artery diseases analyzed the incidence of CAD were more common in male compared to female. Most of the patients were of the age group of 51-60 years (48\%). The prescription pattern of various cardiovascular drugs were found to be as antiplatelet drugs 25 (100\%), antihyperlipidemic drugs 25 (100\%), anti-coagulants 20 (80\%), Nitrates 17(68\%), potassium channel opener 13(52\%), Anti- hypertensives 21 (84\%) The most commonly prescribed drug classes in coronary artery disease were antiplatelet drugs followed by anti hyperlipidemics.A total of 21 (84\%) patients have improved their quality of life after the counseling.

Conclusion: The anti platelets, statins, anticoagulants and beta blockers were apparent in $90 \%$ of prescriptions which shows the rational prescribing behaviour of physicians and also in accordance with AHA/ACC guidelines.
\end{abstract}

Keywords: Coronory artery disease, Observational study, ST segment elevation MI, Non ST segment elevation MI.

\section{Introduction}

Coronory Artery disease (CAD) is the leading cause of morbidity and mortality in both developed and developing countries. It is one of the most common cause of death in India, and its contribution to mortality is rising. In western populations only $23 \%$ of CVD deaths occur before the age of 70 years where as in India, this number is $52 \%$ and mortality occurs more in younger age group than in older age. 
An estimated 17.3 million people died from CVDs in 2008, representing $30 \%$ of all global deaths, Of these an estimated 7.3 million were due to coronary heart disease. By 2030, almost 23.6 million people will die from CVDs, mainly from CAD and stroke. These are projected to remain the single leading causes of death. According to the projections of National Commission and Macroeconomics and Health, Government of India, the total no. of coronary artery disease (CAD) patients in India at the turn of the century was 30 million $(5.3 \%$ of adult population) which could increase to more than 62 million (8.1\%) by the year 2016.A large number of people between 34-64 years die of CVD over the next 30 years as well as an increasing level of morbidity due to CVD.

Cardiovascular disease are a group of general category disease that will affect the heart and the circulatory system. CVD is caused by the disorders of heart, blood vessels that include Coronary Artery Disease (CAD), Congestive Heart Failure (CHF), Stroke, Hypertension, Peripheral Heart Disease and Rheumatic Heart Disease (RHD). Coronory Artery Disease is condition in which there is an inadequate supply of blood and oxygen to a portion of myocardium; it typically occurs when there is an imbalance between myocardial oxygen supply and demand. Atherosclerosis (plaque in artery walls) of the inner lining of the blood vessels that supply blood to the heart is the major cause. CAD begins when bad cholesterol substances (plaques) are deposited within a coronary artery and the plaques narrow the internal diameter of the arteries which may cause a tiny clot to form, which can obstruct the flow of blood to the heart muscle. This reduces the supply of oxygen and nutrients to the heart muscles, which is essential for proper functioning of heart. This may eventually result in a portion of heart being suddenly deprived of its oxygen and leading to death of that area of heart tissue, resulting to heart attack. The risk factors mainly include modifiable and non modifiable factors. High blood pressure, Elevated serum cholesterol, diabetes, Obesity, Sedentary habits, lifestyle changes, Smoking are the modifiable risk factors and age,
Sex and family history are the non modifiable risk factors.

The patient of CAD may present with variety of clinical presentations, such as Chest pain which may be radiating to arms and shoulders, Sweating, Palpitation, Breathlessness or Dyspnoea, Heaviness, Chest discomfort, Weakness etc.. The different CAD subtypes include ST segment elevated myocardial infarction (STEMI), Non-ST segment elevated myocardial infarction (NSTEMI), Unstable Angina, Chronic Stable Angina.

Guidelines based on evidence from randomized controlled trails recommend that antithrombotics, Beta Adrenergic Blockers, Angiotensin Converting Enzyme Inhibitor( ACEI), Angiotensin II receptoblockers (ARBS) and HMGCOA reductase (statins) be used in all patients with symptomatic chronic stable angina or asymptomatic survivors of acute myocardial infarction and following percutaneous coronary intervention or coronary bypass surgery for secondary prevention of myocardial infarction, stroke and death. It has been hypothesized that if used collectively these agents could reduce long term risk of cardiovascular events and mortality.

In developing countries like India the quality of life can be improved by enhancing the standards of medical care at all levels of health care system. To improve the quality of medical care, requires prescribing to be judicious, appropriate, safe, effective and economic. A good prescribing is aim to achieve clinical benefit with minimum risk at cost effective price, while respecting the patient's choice. The analysis of prescription pattern in patients with CAD would help to recognize the current treatment strategies in patients with $\mathrm{CAD}$ having diverse presentation and associated co morbidities. By analyzing the prescription and the medication history the risk factors and trends in prescription can be identified and thereby we can provide appropriate patient counseling and can improve the quality of life of patients with CAD. Short form 36(SF- 36) questionnaires are used to assess the quality of life of patient. It is a 36-item, patientreported survey of patient health. 
This study was therefore planned to scrutinize the prescriptions to determine the appropriateness of the prescriptions and various trends in treatment, so as to provide the basis for modification in the future drug usage in accordance with risk factors and clinical presentations and also assess the improvement of health related quality of life of patients with CAD.

\section{Materials and Methods}

This was a prospective observational study conducted by the Department of pharmacy practice in collaboration with Department of cardiology at a tertiary care hospital in Trivandrum. Institutional ethics committee permission was obtained before initiating the study. Total 25 patients were enrolled in study based on the inclusion and exclusion criteria.

\section{Inclusion Criteria}

- Age 30-80 yrs

- Patients diagnosed with CAD

- Patients who are willing to participate in the study

\section{Exclusion Criteria}

- Age <30 yrs

- CKD patients

- Pregnant women

- Psychiatric patients

- Bleeding disorders/ thrombocytopenia

Written informed consent was obtained from each patient. The procedures followed in the study were in accordance with the ethical standards of Institutional ethics committee. The privacy and the confidentiality of the data was maintained throughout the study.

Total 25 patient's prescriptions were studied and analysed. The demographic data, diagnosis, personal habits and presence of any other co-morbid conditions were recorded from the output prescription sheet or inpatient admission papers. Data related to drugs prescribed was recorded as the name of the drug (Brand name or generic name), dosage form, strength, frequency of dosing, duration of treatment. Similarly the information regarding the availability of the medication and physical examination findings such as BP recordings, ECHO, ECG reporting was noted. Other laboratory investigations carried out within 3 months such as blood sugar level (FBS\& RBS), Lipid profile, serum electrolytes, cardiac markers were also noted. Using a structured interview schedule, information on personal habits, medical history and family history of Hypertension, Diabetes mellitus, Dyslipidemia and coronary risk factors was also recorded.

For this study a questionnaire of the Short Form health Survey (SF-36) has been used to evaluate the HRQoL of the patients with CAD. The questionnaire includes multi-item scales to assess the eight dimensions of wellness; physical functioning, role limitations due to physical health problems, body pain, general health perceptions, social functioning, vitality, energy or fatigue and role limitations due to emotional problems. Each of the subscales is scored on a scale of $0-100$, with high score indicates better HRQoL.

\section{Statistical Analysis}

Frequencies and percentage were calculated as summary measures. Chi-square test for goodness of fit was used for testing significant proportional difference between classes, Chi-square test for association was used for finding significant association between level of diagnosis and different risk factors. If self count $<5$ then Fischer exact test was employed. A calculated $\mathrm{P}$ value $<0.05$ is considered to be statistically significant. All analysis were done with the help of SPSS, version 22.0

\section{Results}

A total of 25 patients who were admitted in the department of cardiology with the diagnosis of CAD were studied.

From the demographic profile, it was observed that among the 25 patients $19(76 \%)$ were males and 6 (24\%) were females. The age distribution of patients with CAD is represented in figure: 1 . 


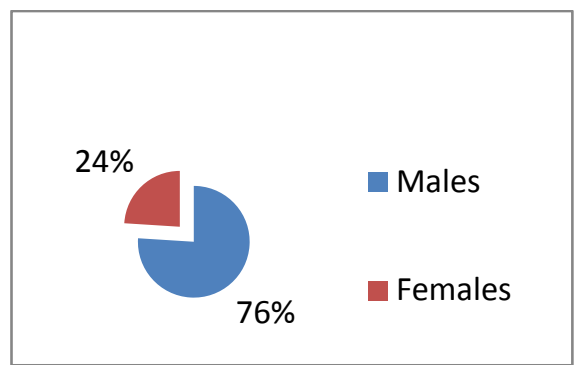

Figure 1 percentage distribution of male and female.

The highest number of patients were between the age group of 51-60 years (12 patients, 48\%) followed by the age group of 61-70 years ( 7 patients, 28\%), 71- 80 yeas (4 patients, 28\%), 71-80 years (4 patients, 16\%) and 41-50 years (2 patients, 8\%). The percentage distribution of age is represented in figure: 2 .

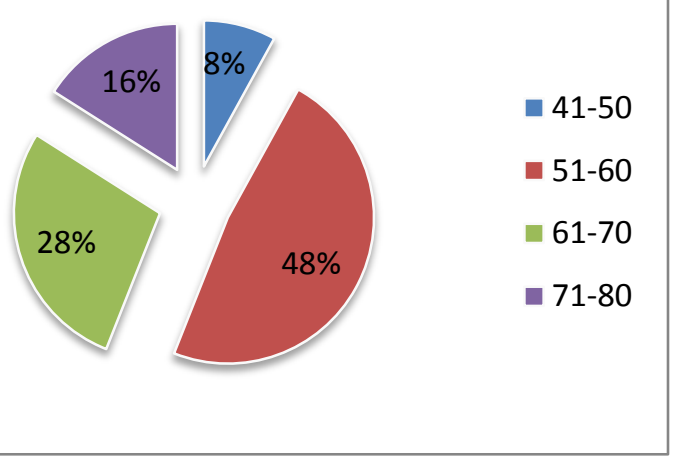

Figure 2; percentage age group of patients Out of the 25 patients, a total of 18 patients (72\%) have undergone both medical treatment and percutaneous coronary interventions, only 4 patients $(16 \%)$ receive drug therapy alone but only 3 patients $(12 \%)$ have undergone CABG. The most common categories of CAD encountered in the study were NSTEMI $(\mathrm{N}=9,64 \%)$ and STEMI $(\mathrm{N}=9$, $36 \%$ ), represented in figure: 3 . No angina were detected during this period.

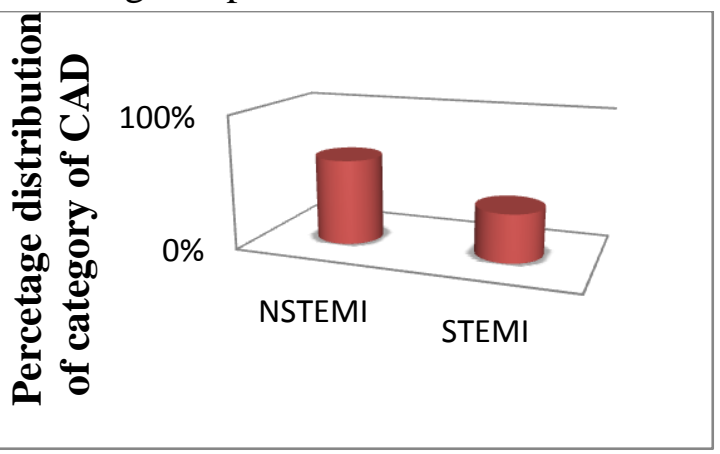

Figure 3 percentage of category of CAD
Table 1

\begin{tabular}{|c|l|c|}
\hline Sl no: & Drugs & $\begin{array}{c}\text { No: of patients \& } \\
\text { percentage distribution }\end{array}$ \\
\hline I. & ANTI PLATELET & \\
\hline & Asprin & $23(92 \%)$ \\
\hline & Clopidogrel & $20(80 \%)$ \\
\hline & Aspirin+ Clopidogrel & $2(8 \%)$ \\
\hline II. & ANTICOAGULANTS & $10(40 \%)$ \\
\hline & Ticagrelol & $8(32 \%)$ \\
\hline & LMWH & $2(24 \%)$ \\
\hline & Fondaparinux & $5(20 \%)$ \\
\hline & Heparin & $17(68 \%)$ \\
\hline III. & NITRATES & \\
\hline & Nitroglycerin & $24(96 \%)$ \\
\hline IV. & STATINS & \\
\hline & Atorvastatin & $14(56 \%)$ \\
\hline V. & K+ CHANNEL OPENER & \\
\hline & Nikorandil & \\
\hline
\end{tabular}

In table 1 chi square test showed that a significant majority of physicians prescribe Aspirin (92\%) followed by Clopidogrel $(80 \%)$. But a significant majority of physicians do not prescribe combination of Aspirin and Clopidogrel. The most commonly prescribed drugs were Atorvastatin (96\%, 24 patients), Aspirin 92\%, 23 patients), Clopidogrel (80\%, 20 patients), Nitrates (68\%, 17 patients), Nikorandil (56\%, 14 patients), Ticagrelol (40\%, 10 patients), Low molecular weight Heparin $(32 \%, 8$ patients) Fondaparinux (24\%, 6 patients) and Heparin (20\%,5patients).

Table 2

\begin{tabular}{c|lc|}
\hline $\begin{array}{l}\text { S1 } \\
\text { no: }\end{array}$ & Drugs & $\begin{array}{c}\text { No: of patients \& } \\
\text { percentage distribution }\end{array}$ \\
\hline I. & BET A BLOCKERS & \\
\hline & Bisoprolo1 & $11(44 \%)$ \\
& Metoprolo1 & $4(16 \%)$ \\
& Nebivolo1 & $2(8 \%)$ \\
\hline II. & ACE INHIBIT ORS & $8(32 \%)$ \\
\hline & Ramipril & $6(24 \%)$ \\
\hline III. & DURETICS & $4(16 \%)$ \\
\hline & Furosemide & $2(8 \%)$ \\
& Eplerenone & $1(4 \%)$ \\
& Aldactone & $2(8 \%)$ \\
& Torsemide & $1(4 \%)$ \\
\hline IV. & Arb s & $1(4 \%)$ \\
\hline & Losartan & \\
\hline & Telmisartan & $1(4 \%)$ \\
& Azilsartan & $1(4 \%)$
\end{tabular}

The most commonly prescribed anti- hypertensives were Bisoprolol followed by Ramipril, Furosemide, Metoprolol, Eplerenone, Nebivolol, Aldactone, 
Losartan, Torsemide, Telmisartan, Azilsartan, Amlodipine, Clinidipine.

The most commonly used category of drugs were Anti platelet and Statins, followed by Anticoagulants, Nitrates, Potassium channel openers, Beta blockers, ACE inhibitors, Diuretics and ARB. Represented in figure:4.

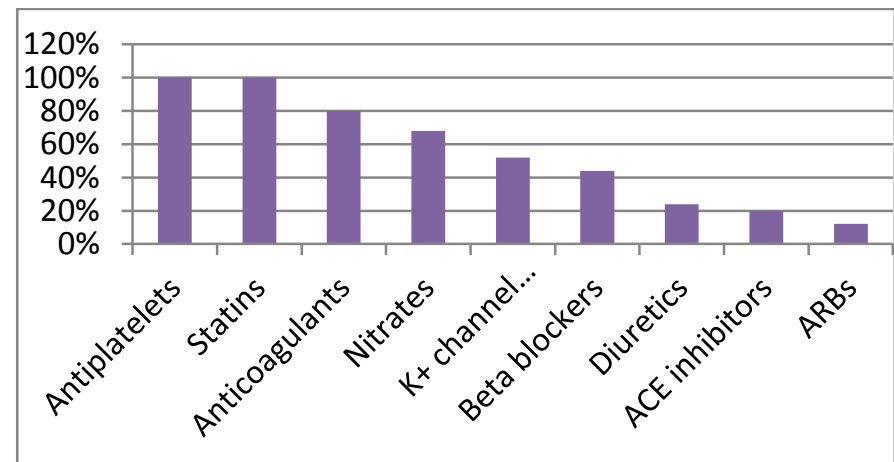

Figure 4: percentage distribution of category of drugs.

The major risk factor found are irregular exercise (80\%), Hypertension (72\%), Diabetes Mellitus (58\%), over weight $(60 \%)$, Dyslipidemia (48\%), family history (40\%), smoking (24\%), Alcoholism $(12 \%)$.

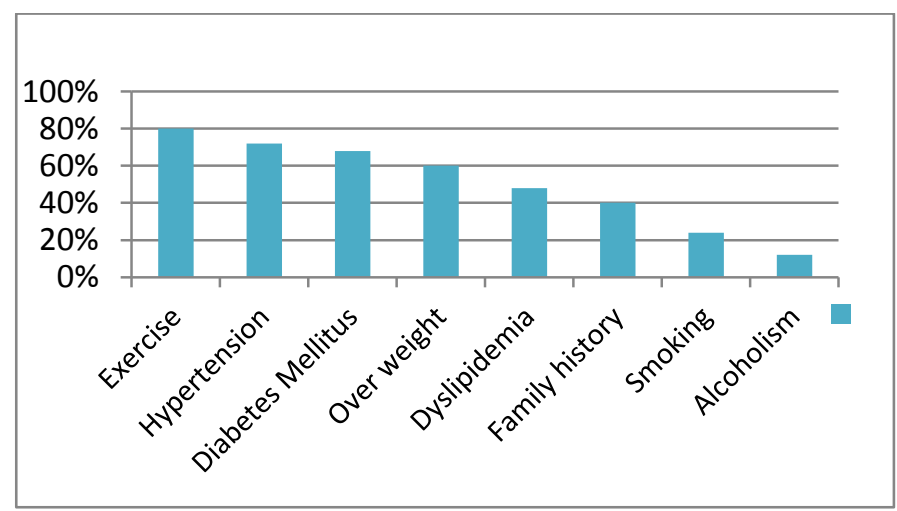

Figure 5; percentage distribution of risk factors

There is significant improvement in the quality of life of patients after counseling $(\mathrm{P}=0.001)$. A total of 21 patients $(84 \%)$ have improved their Quality of life and only 4 patients (16\%) shows no improvement. The patients with stable quality of life belongs to age group of 71-80 years.

\section{Discussion}

The coronary artery disease has become a leading cause of mortality in the south Asian region globally and in India. This study was planned to determine the various treatment strategies involved in the management of coronary artery disease and to assess the changes in the health related quality of life of patients through appropriate interventions involving suitable patient education and life style modification of patients with coronary artery disease.

The WHO recommends the use of aspirin, betablockers and statins in all patients diagnosed with CAD and in addition, ACE inhibitors can be used in patients with left ventricular dysfunction.

Bandla Ashwani et al ${ }^{[1]}$ conducted a study on prescribing pattern of cardiovascular drugs the prescription rate of antiplatelet, anticoagulant and fibrinolytics were $22.46 \%$, followed by $20.07 \%$ of anti-anginal drugs. In our study the most commonly used category of drugs were antiplatelet (100\%) and statins (100\%) followed by anticoagulants (80\%), nitrates $(17 \%)$, potassium channel openers (52\%), beta blockers (44\%), ACEIs (20\%), ARBs (12\%), diuretics (24\%).

Biradar et $\mathrm{al}^{[2]}$ assessed the prescribing pattern in cardiovascular disease. Diuretics (40\%) like furosemide, torsemide, and spirinolactone were used frequently followed by anti-coagulants (30\%) like aspirin and clopidogrel and ramipril was the frequently prescribed ACE inhibitor (20\%).

In our study among the antiplatelets $80 \%$ of the patients received dual antiplatelets therapy (Aspirin and clopidogrel) which shows similarity with Kamah A et al., study in which $90 \%$ of the patients were given dual antiplatelets therapy.

The highest no of patients were in the age group of 51-60 years (48\%), followed by age group of 61-70 years (28\%), 71-80 years (16\%) and $41-50$ years (8\%) which is comparable with the study by Cheah Whyelian et al and Tittu George et al which shows that majority of patients were more than 60 years $(38.6 \%)$ and $51-60$ year age group (39.07\%) respectively. 
Elvin Zengin et $\mathrm{al}^{[4]}$ studied the risk factors of coronary artery disease in the secondary prevention. The major risk factors include diabetes mellitus, hypertension and smoking. In our study the major risk factors include age, gender, smoking, family history were found to be the major risk factors for coronary artery disease.

María Duenas et al assessed the heath related quality of life in coronary patients using SF-36 questionnaire. Several variables related to patients clinical evolution and history of disease have been shown to be related to HRQoL, with worse results among women, subjects with previous history of CHD, and those with another co-morbidity. In this study HRQoL was assessed using SF 36 questionnaire. There is a significant improvement in the quality of life of patients after the counseling. A total of 21 patients have improved their quality of life and only 4 patients (16\%) shows no improvement in quality of life after the counseling. Sunita Dodani ${ }^{[5]}$ conducted a study on the presence of coronary artery disease in diabetic and non diabetic South Asian immigrants. The susceptibility to diabetes among South Asian immigrants promotes an adverse CAD risk.

Joana M Morys et $\mathrm{al}^{[6]}$ assessed the quality of life in patients with coronary heart disease after myocardial infarction and with ischemic heart failure. In patients after MI and with stable CAD anxiety can be reduced by cognitive behavioural therapy while patients with heart failure require long term support therapy to reduce the risk of depressive symptoms.

Anoop Dinesh Shah et al ${ }^{[7]}$ studied type 2 diabetes and incidence of cardiovascular diseases. Heart failure and peripheral arterial disease are the most common initial manifestations of cardiovascular disease in type 2 diabetes mellitus.

Lakshmi Deepika et al conducted a study on cost analysis of oral antiplatelet drugs which showed that a wide variation exist between the maximum and minimum cost among single and combination therapy of oral antiplatelets. In our study a significant majority of physicians do not prescribe combination drug.
Karolina Gierszewska et al ${ }^{[8]}$ assessed the quality of life in patients with coronary artery disease treated with coronary artery bypass grafting and hybrid coronary revascularization. The HRQoL in patients after both modes of revascularization significantly improved after 12 months in all domains. In our study majority of patients received percutaneous coronary intervention and medical management, and only a few received medical management only and a few undergone coronary artery bypass grafting. The quality of life of majority of patients significantly improved.

Hayato Tada et al $^{[9]}$ conducted a study on risk prediction by genetic risk scores for coronary heart disease is independent of self reported family history. The addition of 23 single nucleotide polymorphisms associated with CHD to an existing genetic risk score improved CHD risk prediction. It is particularly useful in young adults. In our study only $40 \%$ of patients had a familial history of coronary artery disease.

Stephen $\mathrm{T}$ Vernon et al conducted a study on increasing proportion of ST elevation myocardial infarction patients with coronary atherosclerosis. The proportion of STEMI patients with STEMI poorly explained by SMuRFs is high, and is significantly increasing.

$\mathrm{N}$ Ershadi Farsani et al conducted a study on the effects of pilates exercise on strength and quality of life in post-coronary artery bypass graft cadiac patients. The pilates exercise improve the strength and quality of life in women with heart disease after coronary artery bypass surgery. Also practicing yoga and meditation are effective methods of stress management and helps to improve the health related quality of life of patients.

\section{Conclusion}

The most commonly used category of drugs were antiplatelet and statins, followed by anticoagulants, nitrates, potassium channel openers, beta blockers, ACE inhibitors, diuretics and ARBs. The most of the drugs were prescribed according to the standard treatment guidelines. There is a significant improvement in the quality of life of patients (84\%) 
through patient education. Several variables related to patients clinical evolution and history with worst results observed with increasing age, subjects with early history of heart diseases and other comorbidities.

\section{References}

1. Bandla Aswani, Purushothama Reddy, P. Yanadaiah, S. Sujatha: A study on prescribing pattern of cardiovascular drugs and potential drug-drug interactions in an inpatient cardiology unit of a cardiac care hospital at Tirupathi; European journal of pharmaceutical and medical research.

2. S M Biradar, Mohammed Naveed, P KeerthiSai, H. PruthviRaju, A Harish Kumar, Anand P Ambali, VijayaSorangavi, B Shivakumar, N V Kalyane: Journal of Drug Delivery and Therapeutics 2018; 8(6s):89-93; http:/ / jddtonline.info.

3. Tittu George Zachariah, Subramanyan K, Pooja M, Vinayak J Kempaller :Drug utilization study in ischaemic heart disease in a tertiary care hospital, Manglore, India; International journal of basic and clinical pharmacology.

4. Elvin Zengin, Christoph Bickel, Renate B Schnabel, Tanja Zeller, Kari J Lackner, Hans J Rupprecht, Stefan Blankenberg, Dirk Westermann, Christoph Sinning for the Athero Gene - Study investigators: Risk factors of Coronary Artery Disease in Secondary Prevention- Results from the Athero Gene- study, PLoS ONE 10(7): e0131434

doi:10.1371/journal pone.0131434.

5. Sunita Dodani, Gyanendra K Sharma: Presence of coronary artery disease in diabetic and non diabetic south asian immigrants; Indian heart journal 70 (2018) 50-55.

6. Joanna M Mory's, jerzybellwon, Stefan hofer, andrzejrynkiewicz, marcingruchala : quality of life in patients with coronary heart disease after myocardial infarction and with ischemic heart failure.

7. Anoop dinesh shah: Type 2 diabetes and incidence of cardiovascular diseases: a cohort study in 1.9 million people; Lancet diabetes endocrinal 2015:3:105-13.

8. Karolina Gierszewska, Izabela Jawoska, Michal Skrzypek, Mariusz Gasior, Robert Pudlo ; Quality of life in patients with coronary artery disease treated with coronary artery bypass grafting and hybrid coronary revascularization. Cardiology journal 2018, Vol. 25, No 5, 621-627

9. HayatoTada, Olle Mlander, Judy Z. Louie, Joeph J. Catanese, Charles M Rowland, James J Devlin, Sekar Kathiresan, Do Shiffman: Risk prediction by genetic risk scores for coronary heart disease is independent of self-reported family history, Euroean Heart Journal(2016) .

10. Dylan L Steen, Irfan khan, Laura Becker, JoAnne M Foody, KatherinaGorcyca, Robert j Sanchez, Robert P Giugliano: Patterns and predictors of lipid- lowering therapy in patients with atherosclerotic cardiovascular disease and/or diabetes mellitus in 2014: Insights from a large US managed - care population,Clinical cardiology 2016.

11. Lipid Research Clinics ProgramThe Lipid Research Clinics Primary Trial results. I. Reduction in incidence of coronary heart disease. JAMA 1984.

12. Blankenhorn DH, Azen SP, Kramsch DM, et al. Coronary angiographic changes with lovastatin therapy: the Monitored Atherosclerosis Regression Study (MARS). Ann Intern Med 1993;119:969-976.

13. Watts GF, Lewis B, Brunt JNH, et al. Effects on coronary artery disease of lipidlowering diet, or diet plus cholestyramine, in the St. Thomas' Atherosclerosis Regression Study (STARS). Lancet1992;339:563-569.

14. Brown G, Albers JJ, Fisher LD, et al. Regression of coronaryartery disease as a result of intensive lipid-lowering therapy in 
men with high levels of apolipoprotein B. $\mathbf{N}$ Engl J Med 1990;323:1289-1298ischman DL, Savage MP, Ellis S, et al. The PalmazSchatz stent. In: Reiber JHC, Serruys PW, eds. Advances in quantitative coronary arteriography. Vol. 137 of Developments in cardiovascular medicine. Dordrecht, the Netherlands: Kluwer Academic, 1993:55366.

15. Fischman DL, Savage MP, Leon MB, et al. Effect of intracoronary stenting on intimal dissection after balloon angioplasty: results of quantitative and qualitative coronary analysis. J Am Coll Cardiol1991;18:14451451. 